\title{
Oil-Loaded Monolinolein-Based Particles with Confined Inverse Discontinuous Cubic Structure (Fd3m)
}

\author{
Anan Yaghmur, ${ }^{\dagger}$ Liliana de Campo, ${ }^{\dagger}$ Stefan Salentinig, ${ }^{\dagger}$ Laurent Sagalowicz,,

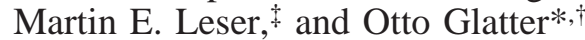 \\ Institute of Chemistry, University of Graz, Graz, Austria, and Nestlé Research Center, \\ Lausanne, Switzerland.
}

Received August 2, 2005. In Final Form: November 8, 2005

\begin{abstract}
In our recent work, we reported on the effect of varying temperature and solubilizing tetradecane (TC) on the structural transitions observed in dispersed particles based on the monolinolein (MLO) - water-TC system. At a given temperature, the addition of TC induces a transition of the internal structure from the bicontinuous cubic phase, Pn3m, to the reversed hexagonal, $\mathrm{H}_{2}$, and to the isotropic liquid phase (water-in-oil (W/O) microemulsions). Our present study focuses on the discovery of a $F d 3 m$ phase (reversed discontinuous micellar cubic), which is formed in the MLOwater-TC system at a specific TC/MLO weight ratio. It is situated between the $\mathrm{H}_{2}$ and the isotropic liquid phase (W/O microemulsion). Remarkably, it is not found in the absence of TC by increasing the temperature. The $F d 3 m$ structure was investigated in detail by means of small-angle X-ray scattering (SAXS), and cryogenic transmission electron microscopy (cryo-TEM). The present work proves that the structural transformation in the dispersed particles from $\mathrm{H}_{2}$ (hexosomes) to the W/O microemulsion system (emulsified microemulsion (EME)) is indirect and it occurs gradually via an emulsified intermediate phase. Specifically, in addition to the nanostructured aqueous dispersions described above, we present new TC-loaded aqueous dispersions with a confined intermediate phase, which is a discontinuous micellar cubic phase of the symmetry $F d 3 \mathrm{~m}$. We denoted this type of emulsified particles as "micellar cubosomes".
\end{abstract}

\section{Introduction}

Self-assembled lyotropic liquid crystalline phases (lamellar, hexagonal, and cubic structures) of surfactant-like lipids and their aqueous nanostructured dispersions have attracted much attention because of their vital role in cell structures and functions. ${ }^{1-6}$ The significant interest in the formulation and the characterization of these systems is driven by both fundamental and practical considerations. ${ }^{7-11}$ They offer many advantages to conventional dispersed systems, such as emulsions or double emulsions ${ }^{12-20}$ because of their possible biological relevance,

* Corresponding author. Tel.: +43 316380 5433. Fax: +43 316380 9850. E-mail: otto.glatter@uni-graz.at.

University of Graz.

Nestlé Research Center.

(1) Hyde, S. T. Curr. Opin. Solid State Mater. Sci. 1996, 1, 653-662.

(2) Seddon, J. M. Curr. Opin. Colloid Interface Sci. 2001, 6, 242-243.

(3) Squires, A. M.; Templer, R. H.; Seddon, J. M.; Woenckhaus, J.; Winter, R.; Finet, S.; Theyencheri, N. Langmuir 2002, 18, 7384-7392.

(4) Larsson, K. Curr. Opin. Colloid Interface Sci. 2000, 4, 449-456.

(5) Almgren, M.; Edwards, K.; Karlsson, G. Colloids Surf. 2000, 174, $3-21$.

(6) Luzzati, V.; Vargas, R.; Mariani, P.; Gulik, A.; Delacroix, H. J. Mol. Biol. 1993, 229, 540-551.

(7) Zhao, D.; Huo, Q.; Feng, J.; Chmelka, B. F.; Stucky, G. D. J. Am. Chem. Soc. 1998, 120, 6024-6036.

(8) Caboi, F.; Nylander, T.; Razumas, V.; Talaikyte,Z.; Monduzzi, M.; Larsson, K. Langmuir 1997, 13, 5476-5483.

(9) Caboi, F.; Amico, G. S.; Pitzalis, P.; Monduzzi, M.; Nylander, T.; Larsson, K. Chem. Phys. Lipids 2001, 109, 47-62.

(10) Caboi, F.; Murgia, S.; Monduzzi, M.; Lazzari, P. Langmuir 2002, 18 , 7916-7922.

(11) Nylander, T.; Mattisson, C.; Razumas, V.; Miezis, Y.; Håkansson, B. Colloids Surf., A 1996, 114, 311-320.

(12) Drummond, C.; Fong, C. Curr. Opin. Colloid Interface Sci. 2000, 4 $449-456$.

(13) Boyd, B. J. Int. J. Pharm. 2003, 260, 239-247.

(14) Siekmann, B.; Bunjes, H.; Koch, M. H. J.; Kirsten, W. Int. J. Pharm. 2002, 244, 33-43.

(15) Landau, E. M.; Rummel, G.; Rosenbusch, J. P.; Cowan-Jacob, S. W. J. Phys. Chem. B 1997, 101, 1935-1937.

(16) Caffrey, M. Curr. Opin. Struct. Biol. 2000, 10, 486-497.

(17) Caffrey, M. J. Struct. Biol. 2003, 142, 108-132.

(18) Yaghmur, A.; de Campo, L.; Sagalowicz, L.; Leser, M. E.; Glatter, O. Langmuir 2005, 21, 569-577. their confined equilibrium nanostructures with high interfacial area, their low viscosity, and their capabilities to solubilize both hydrophilic and hydrophobic active molecules. Therefore, there is a great interest to utilize these dispersions for the administration of drugs, ${ }^{12,19,20}$ for the encapsulation and crystallization of proteins, ${ }^{15-17,21-23}$ or for the formulation of new delivery systems. ${ }^{12,13,19,20}$

Recently, we reported on the effect of varying temperature ${ }^{24}$ and solubilizing tetradecane (TC) ${ }^{18}$ on the reversible structural transitions occurring in the interior of kinetically stabilized monolinolein (MLO)-based particles. Both the increase of temperature at constant oil content and the increase of the oil content in the particles at constant temperature make the spontaneous film curvature $\left(\mathrm{H}_{0}\right)$ of the nanostructure formed inside the particles to be more negative. The formed nanostructures are thermodynamic equilibrium structures, and the emulsified particles show a swelling-deswelling behavior during heating/cooling cycles (in the literature denoted as the "breathing mode" 24 ), indicating that there is a reversible exchange of water inside and outside the confined internal particle structures during the cooling and heating cycles.

The present study describes new results dealing with the elucidation of the structural transitions occurring in TC-loaded dispersed monoglyceride particles. While, in the absence of TC in the MLO-water dispersions, a transition from bicontinuous cubic $(P n 3 m)$ via hexagonal $\mathrm{H}_{2}$ to $\mathrm{L}_{2}$ (water-in-oil (W/O)

(19) Pouton, C. W. Adv. Drug Delivery Rev. 1997, 25, 47-58. 254

(20) Engström, S.; Nordén, T. P.; Nyquist, H. J. Pharm. Sci. 1999, 8, 243-

(21) Rummel, G.; Hardmeyer, A.; Widmer, C.; Chiu, M. L.; Nollert, P.; Locher, K. P.; Pedruzzi, I.; Landau, E. M.; Rosenbuch, J. P. J. Struct. Biol. 1998, 121, $82-91$.

(22) Angelova, A.; Angelov, B.; Papahadjopoulos-Sternberg, B.; Ollivon, M.; Bourgaux, C. Langmuir 2005, 21, 4138-4143.

(23) Misquitta, L. V.; Misquitta, Y.; Cherezov, V.; Slattery, O.; Mohan, J. M.; Hart, D.; Zhalnina, M.; Cramer, W. A.; Caffrey, M. Structure 2004, 12, $2113-$ 2124.

(24) de Campo, L.; Yaghmur, A.; Sagalowicz, L.; Leser, M. E.; Watzke, H.; Glatter, O. Langmuir 2004, 20, 5254-5261. 
microemulsion) phases was observed upon temperature increase, ${ }^{24}$ a slightly different transition scheme is occurring upon the addition of TC to the MLO-water dispersion at constant temperature. ${ }^{18}$ Our present small-angle X-ray scattering (SAXS) and cryogenic transmission electron microscopy (cryo-TEM) results suggest that the transition between the hexosomes $\left(\mathrm{H}_{2}\right)$ and the emulsified microemulsion (EME) particles is not occurring directly (as observed in the binary MLO-water particle system when temperature is increased ${ }^{24}$ ) but via the formation of an intermediate type of particle structure. As we will discuss below, this intermediate phase turned out to be a discontinuous micellar cubic phase of the symmetry $F d 3 m$. We denoted this type of dispersed mesophase particles as "micellar cubosomes".

To better understand the phase transitions occurring in the interior of the mesophase particles, it is necessary to compare the findings with the phase behavior observed in the corresponding bulk (nondispersed) system. Our recent work on the phase diagram of the binary bulk MLO/water system ${ }^{24}$ did not reveal any evidence for the existence of a discontinuous micellar cubic phase of the symmetry $F d 3 m$ by varying the temperature from 20 to $95^{\circ} \mathrm{C}$. This correlates also with findings reported in other previous studies on the binary phase diagram of various monoglycerides/water and phospholipids/water mixtures. $3,16,17,25-28$ However, as shown in the present work, the addition of TC to the bulk MLO-water system induced the appearance of the $F d 3 m$ cubic bulk phase in the MLO-water system. This is a quite intriguing result because, in the literature, only a limited number of similar systems was described so far, where the formation of the bulk $F d 3 m$ cubic phase is occurring. For instance, Seddon and co-workers ${ }^{29-32}$ mentioned the formation of the $F d 3 m$ cubic phase in the ternary system phosphatidylcholine/ diacylglycerols/water ${ }^{30,32}$ and in the binary system glycolipid/ water. ${ }^{30,31}$ Nakano et al. ${ }^{33}$ found a (dispersed) $F d 3 m$ cubic phase based on a monoolein (MO)/oleic acid mixture.

\section{Materials and Methods}

Materials. MLO (emulsifier TS-PH 039, glycerol monolinoleate) was supplied by Danisco A/S (Brabrand, Denmark). This distillated monoglyceride consists of $93.8 \%$ monoglyceride and $4.1 \%$ diglycerides. The fatty acids are composed of $91.8 \%$ linoleate, $6.8 \%$ oleate and about $1 \%$ saturated fatty acids. TC was obtained from Sigma Chemical Co. (St. Louis, Missouri, USA). Pluronic F127 $\left(\mathrm{PEO}_{99}-\mathrm{PPO}_{67}-\mathrm{PEO}_{99}\right.$ ) was a gift from BASF Corporation (Mount Olive, New Jersey, USA). These ingredients were used without further purification. The water was double-distilled.

The nondispersed samples were prepared in Pyrex tubes by weighing the appropriate amounts of MLO, TC, and water, heating them by using an air gun, and homogenizing them by vigorous agitation with a vortex. Then the mixtures were cooled to room temperature $\left(\sim 25^{\circ} \mathrm{C}\right)$. To ensure thermodynamic equilibrium of the samples, they were frozen for at least $1 \mathrm{~h}$ prior to SAXS investigations $^{34}$ and then equilibrated for some hours at room temperature.

(25) Lutton, E. S. J. Am. Oil Chem. Soc. 1965, 42, 1068-1070.

(26) Caffrey, M. Biophys. J. 1989, 55, 47-52.

(27) Mezzenga, R.; Grigorov, M.; Zhang, Z.; Servais, C.; Sagalowicz, L.; Romoscanu, A. I.; Khanna, V.; Meyer, C. Langmuir 2005, 21, 6165-6169.

(28) Mezzenga, R.; Meyer, C.; Servais, C.; Romoscanu, A. I.; Sagalowicz, L.; Hayward, R. C. Langmuir 2005, 21, 3322-3333.

(29) Duesing, P. M.; Templer, R. H.; Seddon, J. M. Langmuir 1997, 13, 351359.

(30) Seddon, J. M.; Robins; J.; Gulik-Krzywicki, T.; Delacroix, H. Phys. Chem. Chem. Phys. 2000, 2, 4485-4493.

(31) Seddon, J. M.; Zeb, N.; Templer, R. H.; McElhaney, R. N.; Mannock, D. A. Langmuir 1996, 12, 5250-5253.

(32) Seddon, J. M. Biochemistry 1990, 29, 7997-8002.

(33) Nakano, M.; Teshigawara, T.; Sugita, A.; Leesajakul, W.; Taniguchi, A.; Kamo, T.; Matsuoka, H.; Handa, T. Langmuir 2002, 18, 9283-9288.

(34) Seddon, J. M.; Templer, R. H. In Handbook of Biological Physics; Hoff, A. J., Ed.; Elsevier SPC: Amsterdam, 1995; Vol. 1, p 97.
Table 1. Reflection Laws for Space Groups that Have Been Determined for Lamellar, Cubic, and Hexagonal Phases Containing Membrane Lipids
$\mathrm{L}_{\alpha}$
$\left(\frac{a}{d}\right)=1,2,3,4,5,6 \ldots$ with $d=2 \pi / q, a$...lattice parameter
$\operatorname{Pn} 3 m\left(\mathrm{C}_{\mathrm{D}}\right)\left(\frac{a}{d_{\mathrm{hkl}}}\right)^{2}=2,3,4,6,8,9,10,11 \ldots$
$\operatorname{Ia3d}\left(\mathrm{C}_{\mathrm{G}}\right) \quad\left(\frac{a}{d_{\mathrm{hkl}}}\right)^{2}=6,8,14,16,20,22,24 \ldots$
$\operatorname{Im} 3 m\left(\mathrm{C}_{\mathrm{P}}\right)\left(\frac{a}{d_{\mathrm{hkl}}}\right)^{2}=2,4,6,8,10,12,14 \ldots$
$P m 3 n$
$\left(\frac{a}{d_{\mathrm{hkl}}}\right)^{2}=2,4,5,6,8, \ldots$
$F d 3 m$
$\left(\frac{a}{d_{\mathrm{hkl}}}\right)^{2}=3,8,11,12,16,19,24 \ldots$
$\mathrm{H}_{2}$ $\left(\frac{\sqrt{3} \cdot a}{2 \cdot d_{\mathrm{hk}}}\right)^{2}=1,3,4,7$

For the formation of MLO-based oil-loaded aqueous dispersions, a mixture of MLO (viscous liquid) and TC was weighed at $25^{\circ} \mathrm{C}$ into a $20 \mathrm{~mL}$ vial. F127 and then water was added to yield a sample with a total weight of $10 \mathrm{~g}$. Then the sample containing all four ingredients was treated by ultrasonication for $20 \mathrm{~min}$, resulting in a milky dispersion. The typical composition of the prepared dispersions was 95 wt \% water and 5 wt \% MLO/oil/F127 mixture, whereby the F127 concentration was kept constant, that is, 0.375 wt $\%$ (if not reported otherwise) and the weight ratio $\alpha(\alpha=[$ (mass of TC)/(mass of MLO)] 100) was varied. The value of $\alpha$ was in the range of $0-60$. Ultrasonication was carried out using a high-intensity ultrasonic processor (SY-LAB GmbH, Pukersdorf, Austria), at 30\% of the maximum power, and $0.5 \mathrm{~s}$ pulses interrupted by $0.5 \mathrm{~s}$ breaks. No external sample cooling was applied.

The structure of the dispersed and nondispersed samples was determined by SAXS technique and cryo-TEM. The SAXS equipment used consisted of a SAXSess camera (Anton-Paar, Graz, Austria), which was connected to an X-ray generator (Philips, PW 1730/10) operating at $40 \mathrm{kV}$ and $50 \mathrm{~mA}$ with a sealed-tube $\mathrm{Cu}$ anode. A Göbel mirror was used to convert the divergent polychromatic X-ray beam into a focused line-shaped beam of $\mathrm{Cu} K \alpha$ radiation $(\lambda=0.154 \mathrm{~nm})$. The two-dimensional scattering pattern is recorded by an imagingplate detector (model Fuji BAS1800 from Raytest, Straubenhardt, Germany) and integrated to the one-dimensional scattering function $I(q)$ using SAXSQuant software (Anton Paar, Graz, Austria), where $q$ is the length of the scattering vector, defined by $q=(4 \pi / \lambda) \sin \theta / 2$, $\lambda$ is the wavelength, and $\theta$ is the scattering angle. For indexing the different mesophases, we used the reflection laws summarized in Table 1. These comprise the space groups that have been determined for cubic and hexagonal phases containing membrane lipids. ${ }^{35}$ The interplanar distances $d$ between 2 reflecting planes are given by $d$ $=2 \pi / q$, which enables us to calculate the corresponding mean lattice parameter $a$. The scattering profiles of the so-called $\mathrm{L}_{2}-$ phase show only one broad correlation peak, for which the position of the observed maximum is shifted to lower q-values due to the "smearing effects" of the line-shaped primary beam. These profiles were desmeared by fitting these data with the generalized indirect Fourier transformation (GIFT) method. ${ }^{36}$ For the $\mathrm{L}_{2}-$ phase, $d$ is called characteristic distance.

The sample was filled at $25^{\circ} \mathrm{C}$ into the sample holder (capillary in a metal block, temperature controlled by a Peltier element, \pm 0.1 ${ }^{\circ} \mathrm{C}$ ) and equilibrated at each experimental temperature for at least 10 min before measurement. All temperature scans were performed in the heating direction. The nondispersed and dispersed samples were exposed for 10 and $45 \mathrm{~min}$, respectively.

(35) Lindblom, G.; Rilfors, L. Biochim. Biophys. Acta 1989, 998, 221-256. 1216 

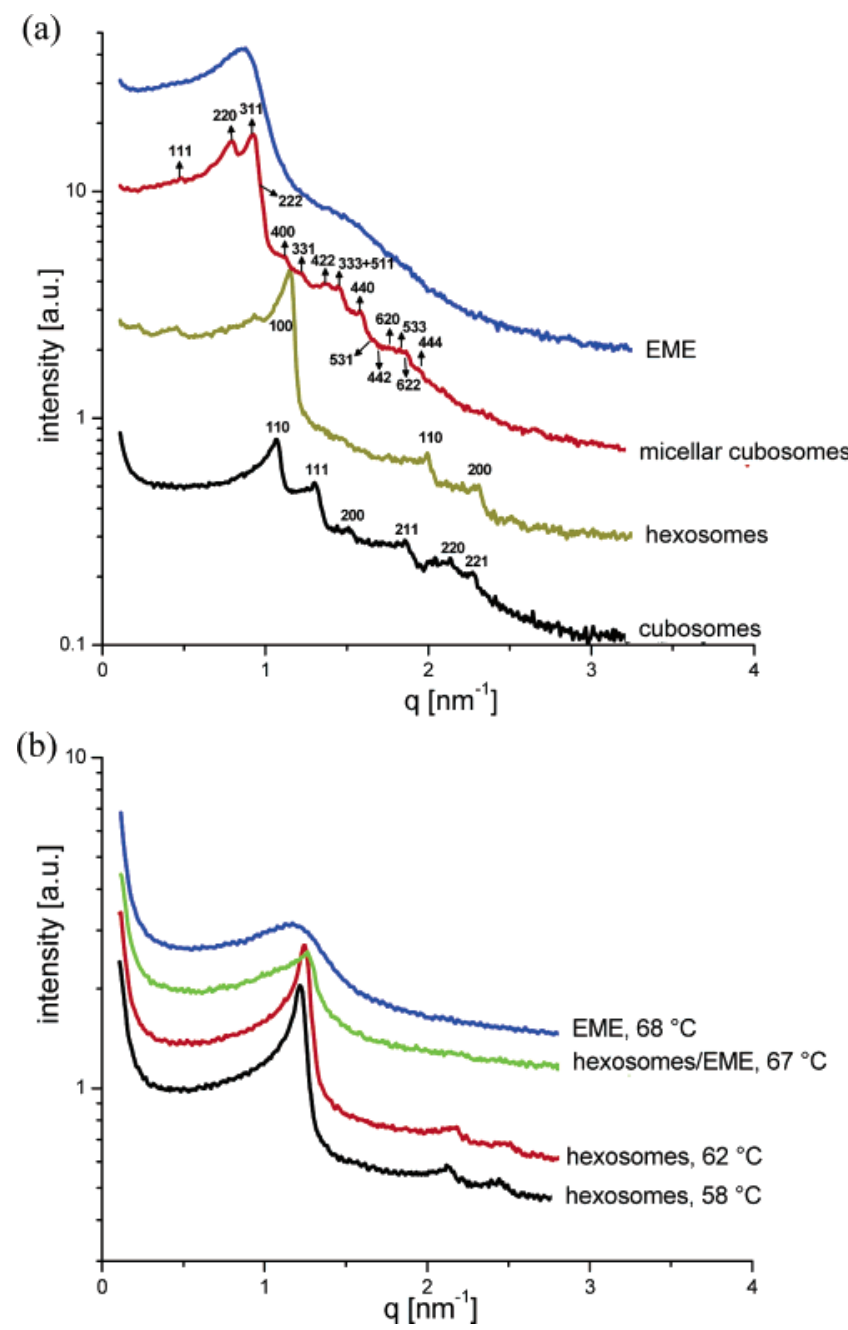

Figure 1. (a) Effect of solubilized TC content on the scattering curves of MLO-based emulsified systems at $25{ }^{\circ} \mathrm{C}$. In these dispersions, the $\alpha$ ratio [\{(mass of oil $) /($ mass of MLO $)\} \cdot 100]$ varies from 0 (cubosomes), to 28 (hexosomes), 40 (micellar cubosomes), and 60 (EME). All peaks in the scattering curves are identified by their Miller indices. (b) Temperature dependence of scattering curves for the TC-loaded dispersion with $\alpha=19$. The direct temperatureinduced hexosomes-EME transition occurs at about $68^{\circ} \mathrm{C}$. The curves are shifted by a constant arbitrary factor for better visibility.

Cryo-TEM was performed as described in our previous works. ${ }^{18,24,37}$ The humidity in the environmental chamber was $100 \%$.

\section{Results and Discussion}

The present work deals with the impact of TC solubilization on the internal nanostructure of MLO-based aqueous dispersions stabilized by the polymer F127. Figure 1a shows the SAXS scattering curves obtained from dispersions in which the $\alpha$ value was varied from 0 to 60 at $25^{\circ} \mathrm{C}$. In the absence of oil $(\alpha=0)$, the scattering curve of the dispersion shows six peaks in the characteristic ratio for a cubic structure of the type $P n 3 m$, denoted by the number $\mathrm{Q}^{224}$ (cubosomes, emulsified cubic phase (ECP)). For the TC-loaded dispersion with $\alpha=28$, the scattering curve of the dispersion shows three peaks in the characteristic ratio for a hexagonal phase (hexosomes, EHP). Intriguingly, a further increase of the TC concentration in the dispersion $(\alpha=40)$ gives rise to a scattering curve that shows more than seven peaks. These peaks are in the characteristic ratio for a discontinuous

(37) Sagalowicz, L.; Michel, M.; Adrian, M.; Frossard, P.; Rouvet, M.; Watzke H. J.; Yaghmur, A.; de Campo, L.; Glatter, O.; Leser, M. E. J. Microsc., in press.
Table 2. Structure Parameters ${ }^{a}$ as Derived from SAXS Investigations that Were Carried out on the Dispersed Systems as a Function of $\alpha^{b}$

\begin{tabular}{cccclcr}
\hline $\begin{array}{c}\text { investigated } \\
\text { systems }\end{array}$ & $\begin{array}{c}\text { temperature } \\
\left({ }^{\circ} \mathrm{C}\right)\end{array}$ & $\begin{array}{c}\text { water } \\
(\text { wt } \%)\end{array}$ & a & $\begin{array}{c}\text { space } \\
\text { group }\end{array}$ & $\begin{array}{c}\text { mean } a \\
(\mathrm{~nm})\end{array}$ & $\begin{array}{c}d \\
(\mathrm{~nm})\end{array}$ \\
\hline Figure 1a & 25 & 95 & 0 & $P n 3 m$ & 8.3 & \\
& 25 & 95 & 28 & $\mathrm{H}_{2}$ & 6.3 & \\
& 25 & 95 & 40 & $F d 3 m$ & 21.7 & \\
& 25 & 95 & 60 & $\mathrm{~L}_{2}$ & & 6.9 \\
Figure 1b & 58 & 95 & 19 & $\mathrm{H}_{2}$ & 5.9 & \\
& 62 & 95 & 19 & $\mathrm{H}_{2}$ & 5.8 & \\
& 67 & 95 & 19 & $\mathrm{H}_{2} / \mathrm{L}_{2}$ & 5.8 & $\sim 5.0$ \\
Figure 2a & 68 & 95 & 19 & $\mathrm{~L}_{2}$ & & 4.9 \\
& 25 & 0 & 50 & $\mathrm{~L}_{2}$ & & 3.6 \\
& 25 & 5 & 50 & $\mathrm{~L}_{2}$ & & 4.4 \\
& 25 & 10 & 50 & $\mathrm{~L}_{2}$ & & 5.1 \\
& 25 & 17 & 50 & $\mathrm{~L}_{2}$ & & 6.2 \\
& 25 & 20 & 50 & $F d 3 m$ & 21.7 & \\
Figure 2b ${ }^{c}$ & 25 & 25 & 50 & $F d 3 m$ & 22.0 & \\
& 25 & 95 & 50 & $F d 3 m$ & 22.0 & \\
& 25 & $20(95)^{d}$ & 50 & $F d 3 m$ & 22.0 &
\end{tabular}

${ }^{a}$ The mean lattice parameter, $a$, for the cubic and the hexagonal phases and the $d$ spacing that is called the characteristic distance for the $\mathrm{L}_{2}-$ Phase. ${ }^{b}$ The aqueous dispersions contain $95 \mathrm{wt} \%$ water and 5 wt $\%$ of a mixture of MLO, TC, and F127. ${ }^{c}$ Samples in Figure $2 b$ represent SAXS data for both systems (the dispersed and the nondispersed mesophases with excess water). They have the same space group and same structure parameters. ${ }^{d}$ The water content in the emulsified samples (95 wt \%).

\section{Scheme 1. Possible Internal Nanostructures of MLO-Based Emulsified Droplets}

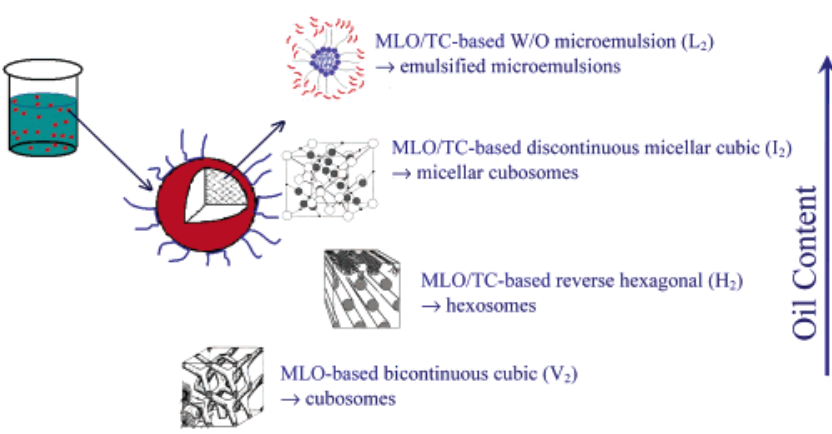

micellar cubic structure of the type $F d 3 m$, which is denoted by the number $\mathrm{Q}^{227}$ (micellar cubosomes, emulsified micellar cubic phase (EMCP)). As presented in Table 2, the mean lattice parameter, $a$, of the cubosomes, hexosomes, and micellar cubosomes are 8.3, 6.3, and $21.7 \mathrm{~nm}$, respectively. Our results indicate that the structural transformation from hexosomes to EME is not direct, but takes place in a narrow TC concentration range in which micellar cubosomes with the internal space group $F d 3 m$ (denoted also by the symbol "I $\mathrm{I}_{2}$ ") are formed. The $F d 3 m$ phase appears between the $\mathrm{H}_{2}$ and W/O microemulsion phase. At higher TC concentrations ( $\alpha \geq 60)$, the scattering curve shows only one broad peak. This is typical for a concentrated microemulsion phase. The appearance of a shoulder around $q=$ $1.5-2.0 \mathrm{~nm}^{-1}$ for these systems in addition to the first broad peak can be explained by the fact that the solubilized water increases the order in the inverse microemulsion system. This leads to the appearance of a side maximum in the scattering curves, which is directly related to the main maximum and therefore does not indicate the presence of any additional phase in the system.

Scheme 1 summarizes the transitions occurring when TC is added to a MLO/water dispersion. As shown in the scheme, self-assembled nanostructures, such as inverted-type mesophases 
of the bicontinuous cubic of the symmetry $P n 3 m$ or the micellar cubic of the symmetry $F d 3 m$, the hexagonal geometry, or the reverse microemulsion phases, can be dispersed using a polymeric stabilizer, such as the triblock copolymer Pluronic F127. At constant temperature, increasing TC content in the MLO-waterF127 system leads to the transformation of the internal nanostructure of the kinetically stabilized particles from a $P n 3 m$ (cubosomes) to a $\mathrm{H}_{2}$ (hexosomes), to an $F d 3 m$ (micellar cubosomes), and to a W/O $\left(\mathrm{L}_{2}\right)$ microemulsion phase (EME). Nakano et al. ${ }^{33}$ reported on a similar phase transition event $\left(P n 3 m-\mathrm{H}_{2}\right.$-inverted micellar cubic phase with the $\mathrm{Fd} 3 m$ space group) occurring in MO-based aqueous dispersions stabilized by F127 when oleic acid is added to the system. However, they did not observe the transition from the $F d 3 m$ to the W/O microemulsion phase. Even when MO is replaced completely by oleic acid, dispersed $\mathrm{Fd} 3 \mathrm{~m}$ and no EME particles are formed. In our case, the complete replacement of MLO by TC (MLOfree sample) leads to the formation of "structureless" particles (normal emulsions). ${ }^{18}$

It is interesting to note that increasing temperature in the hexosome dispersion loaded with a small amount of TC does not induce the formation of the $F d 3 m$ phase, but rather a direct transition from $\mathrm{H}_{2}$ to W/O microemulsion. A typical example for this direct structural transition is presented in Figure 1b. Increasing temperature at constant oil content is expected to cause a decrease in the hydration of the MLO headgroup and simultaneously cause an increase in the kink states in the lipid acyl chains and thus their effective volume. ${ }^{18,38}$ This means that, in this case, an increase $\mathrm{e}^{18,24}$ of the critical packing parameter (CPP) leads directly to the formation of inverse discrete microemulsion droplets, which are embedded in a continuous hydrophobic matrix (the oil phase). There is no evidence that a temperature increase induces the microemulsion droplets to pack first on a $F d 3 m$ cubic lattice before the isotropic microemulsion phase is formed.

The $F d 3 m$ cubic phase consists of a three-dimensional periodically ordered complex packing of two different types of discrete inverse micelles, both quasi-spherical. ${ }^{30-32,39}$ In this phase, the unit cell contains 8 larger inverse micelles of symmetry $43 \mathrm{~m}$ arranged tetrahedrally on a diamond lattice, and 16 smaller reverse micelles of symmetry $\overline{3} \mathrm{~m}$. These discrete aggregates, with a core consisting of hydrophilic aqueous domains, are embedded in a continuous three-dimensional hydrophobic matrix (the oil phase).

To better understand the dispersed $F d 3 m$ systems, it is important to compare the internal structure of the dispersions to that of the corresponding bulk (nondispersed) ternary MLO/TC/water system. Figure $2 \mathrm{a}$ shows the effect of hydration on the structure of bulk TC/MLO mixtures at $\alpha=50$ : an inverse microemulsion system is formed with increasing water content $(0-17$ wt \% water; scattering curves of these systems have a single broad peak), and a $F d 3 m$ phase is formed at higher water contents (20-25 wt \% water). Thereby, with increasing water content in the single-phase regions, the observed peaks move to lower angles, corresponding to larger structures ("swelling" with water). Above $25 \%$ water, the water solubilization capacity of this sample is exceeded, and the $F d 3 m$ phase is in equilibrium with excess water. Above this fully hydrated condition, no more change is observed in the peak positions and in the corresponding structure parameters. The scattering curves of each of the micellar cubosomes correspond well to those of the nondispersed fully hydrated bulk $F d 3 m$ phases with the same $\alpha$-value (see Figure

(38) Geil, B.; Feiweier, T.; Pospiech, E. M.; Eisenblatter, J.; Fujara, F.; Winter, R. Chem. Phys. Lipids 2000, 106, 115-126.

(39) . Luzzati, V.; Vargas, R.; Gulik, A.; Mariani, P.; Seddon, J. M.; Rivas, E. Biochemistry 1992, 31, 279-285.
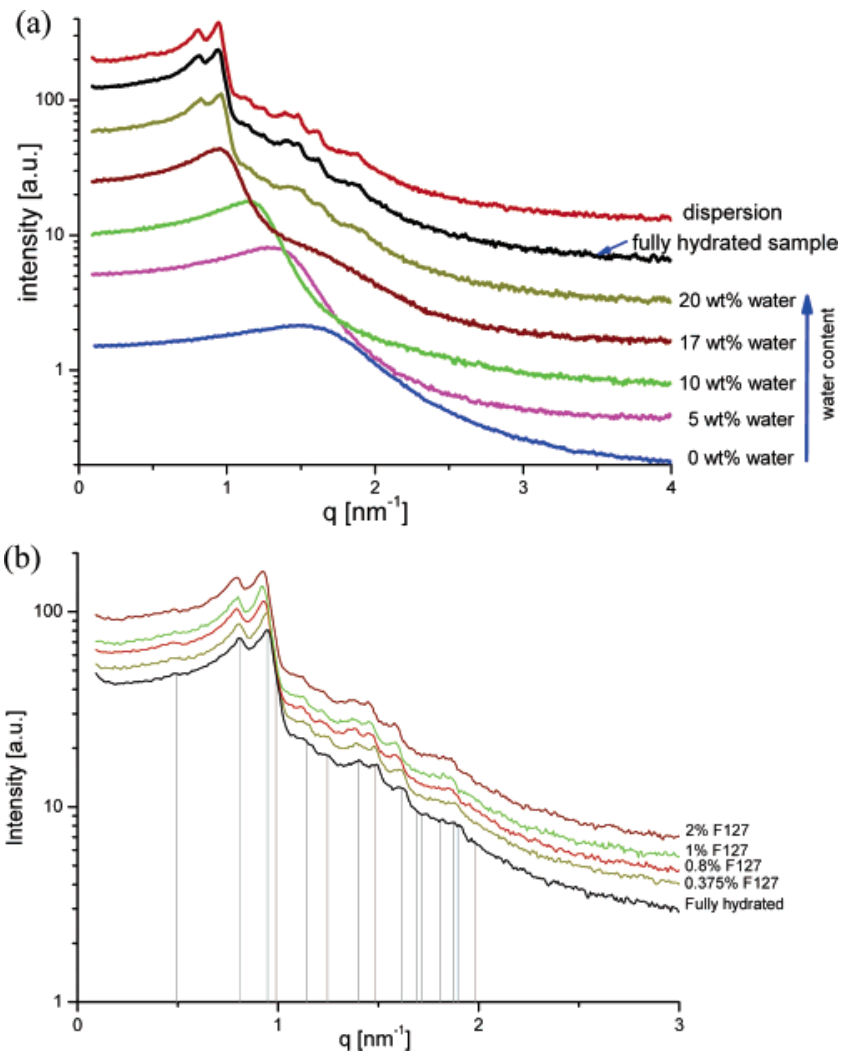

Figure 2. (a) Comparison of scattering curves from the TC-loaded micellar cubosomes with $\alpha=50$ (red line) with those from the corresponding nondispersed bulk sample with varying water content at $25^{\circ} \mathrm{C}$. (b) Effect of $\mathrm{F} 127$ concentration on the scattering curves of the TC-loaded micellar cubosomes with $\alpha=50$. The stabilizer concentration varies from 0.375 to $2 \mathrm{wt} \%$. The curves are shifted by a constant arbitrary factor for better visibility. The vertical lines show the position of the peaks for the $F d 3 m$ structure, as indexed in Figure 1a for the micellar cubosomes.

2a). The mean lattice parameter $a$, for both dispersed and nondispersed $F d 3 m$ phase, is $22.0 \mathrm{~nm}$ (see Table 2). These results indicate that the self-assembled inner particle $F d 3 m$ nanostructure is preserved during the dispersion procedure. This implies that F127 is an efficient stabilizer and does not influence the internal $\mathrm{Fd} 3 \mathrm{~m}$ structure. As shown in Figure 2b, increasing F127 concentration from 0.375 to $2 \mathrm{wt} \%$ does not influence the internal $\mathrm{Fd} 3 \mathrm{~m}$ structure. Our results confirm previous reports on the effect of F127 on the stabilization of hexosomes and micellar cubosomes. ${ }^{18,33,40}$

For the TC-loaded dispersion with $\alpha=40$, the cryo-TEM image, shown in Figure 3, reveals the formation of dispersed lipidic particles. These particles show a periodic internal motif. The fast Fourier transform (FFT, inset of Figure 3) shows a 6-fold symmetry, and therefore the electron beam is very likely parallel to the [111] direction. The interplanar distances corresponding to the intensity peak in the FFT is about $8 \mathrm{~nm}$, which is in agreement with the presence of $\{220\}$ reflections leading to a lattice parameter of about $22 \mathrm{~nm}$, confirming the SAXS analysis. Precise indexation of the FFT spots is performed the same way in which indexation of electron diffraction pattern is performed, ${ }^{41}$ considering both angles between spots and corresponding interplanar distances. In addition to the $\{220\}$ reflection, the weak $\{111\}$, the $\{311\}$, and the $\{222\}$ reflections are also

(40) Gustafsson, J.; Ljusberg-Wahren, H.; Almgren, M.; Larsson, K. Langmuir 1997, 13, 6964-6971

(41) Edington, J. W. Practical Electron Microscopy in Materials Science Phillips: Eindhoven, The Netherlands, 1974; Vol. 2. 


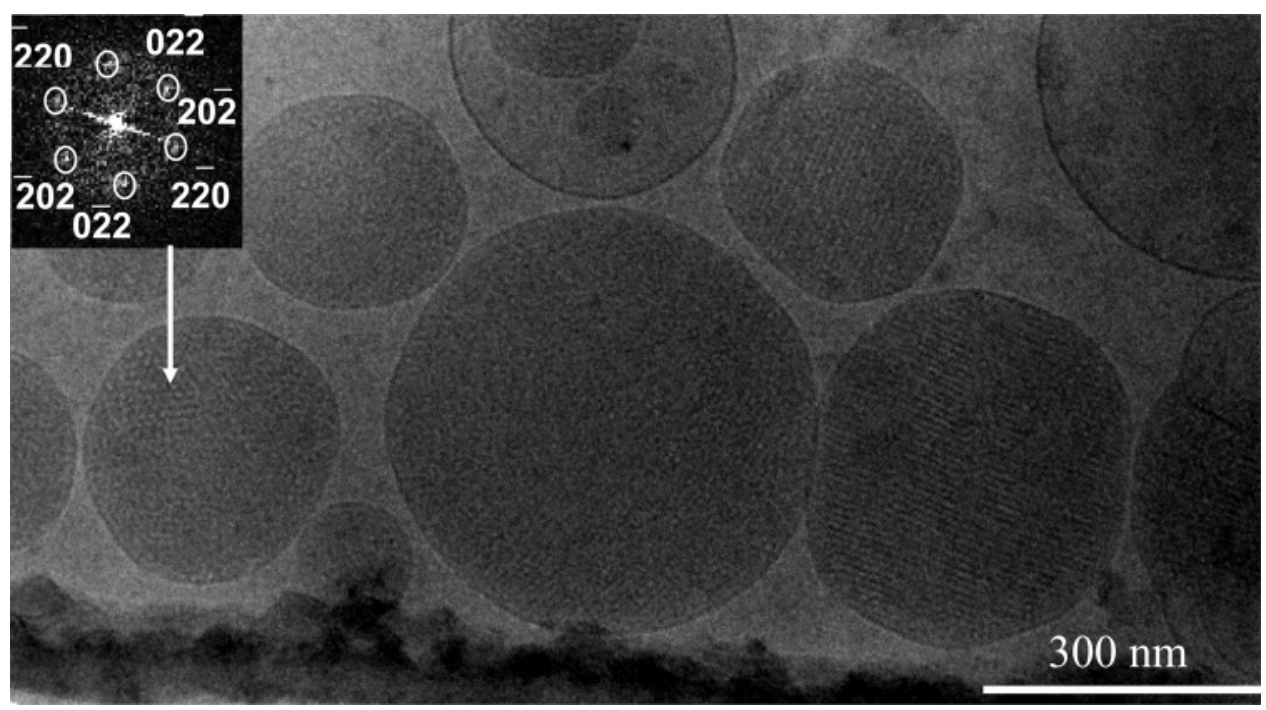

Figure 3. (a) Cryo-TEM image of MLO-based aqueous TC-loaded dispersion with $\alpha=40$. In this image, emulsified dispersed micellar cubosomes with an internal $F d 3 m$ phase are observed. Inset is an FFT of the arrowed particle viewed along [111]. A hexagonal motif formed by the $\{220\}$ reflections is observed.

observed in other particles. The contrast of micellar cubosomes is somehow similar to the contrast obtained for "normal" cubosomes. However, in micellar cubosome dispersions, no vesicles are associated with the particles. This is different for normal cubosomes with a confined bicontinuous cubic phase, which are often connected to vesicles. ${ }^{24}$

In summary, the self-assembled nanostructure of the $F d 3 m$ discontinuous micellar cubic phase is formed when mixing MLO, water, and TC together in the appropriate proportion. Addition of TC to dispersed MLO-based particles induces the transformation from bicontinuous cubic $(P n 3 m)$ via $\mathrm{H}_{2}$ to discontinuous micellar cubic $(F d 3 m)$ and to $\mathrm{L}_{2}$ (W/O microemulsion). All dispersions were stabilized by the polymer F127. Interestingly enough, the $F d 3 m$ cubic phase is not formed when the system is heated. A temperature increase changes the internal nanostructure of the particles from a $\mathrm{Pn} 3 \mathrm{~m}$ (cubosomes), to a $\mathrm{H}_{2}$ (hexosomes) directly to the W/O $\left(\mathrm{L}_{2}\right)$ microemulsion phase (EME). Temperature increase does not induce the formation of an $F d 3 m$ cubic phase between the $\mathrm{H}_{2}$ and $\mathrm{L}_{2}$ region. A possible reason for this is certainly related to the severe packing constraints appearing in the $F d 3 m$ cubic phase. ${ }^{29-31}$ These constraints are the result of the competition between the constraints related to packing (the need to uniformly fill the hydrophobic volume with the fluid hydrocarbon chains) and the desire to maintain a uniform mean interfacial curvature. Our results should trigger new fundamental work aimed at understanding the principles leading to the formation of the reversed $F d 3 m$ liquid crystalline mesostructure.

Acknowledgment. The Austrian Science Fund FWF (M711N03) and the Nestlé Research Center, Lausanne, Switzerland financially supported this work. We thank Martin Michel and Heribert Watzke (Nestlé Research Center, Lausanne, Switzerland) for helpful discussions and critical reading. We also thank Fred Chavannes, Olivier Girault, Jean-Albert Stucki, Dino Tomasi, and Laurent Peissard (CRN) for having built the environmental chamber for the cryo-TEM analysis. Thanks to Günther Scherf (IFC) for technical help.

\section{LA052109W}

